\title{
Cholesterol High, CTCAE
}

National Cancer Institute

\section{Source}

National Cancer Institute. Cholesterol High, CT CAE. NCI Thesaurus. Code C143368.

A finding based on laboratory test results that indicate higher than normal levels of cholesterol in a blood specimen. 